\title{
Therapeutic patient education in oncology: pedagogical notions for women's health and prevention
}

M. RIZZO, MD, PHD, Department of Clinical Medicine and Emerging Diseases, University of Palermo, Palermo, A. MIGNECO, Bsc, Department of Clinical Medicine and Emerging Diseases, University of Palermo, Palermo, P. MANSUETO, MD, Department of Clinical Medicine and Emerging Diseases, University of Palermo, Palermo, G. TRINGALI, MD, Department of Public Health, University of Palermo, Palermo, G. DI LORENZO, MD, Department of Clinical Medicine and Emerging Diseases, University of Palermo, Palermo, \& G.B. RINI, MD, Department of Clinical Medicine and Emerging Diseases, University of Palermo, Palermo, Italy

RIZZO M., MIGNECO A., MANSUETO P., TRINGALI G., DI LORENZO G. \& RINI G.B. (2007) European Journal of Cancer Care 16, 9-11

Therapeutic patient education in oncology: pedagogical notions for women's health and prevention

Therapeutic patient education has been defined by the World Health Organization as a comprehensive approach to support patients and their families to better understanding of their diseases. In oncology, the contribution of therapeutic education may enable the patients to have adequate information of the illness, to actively participate in the management of the disease, to understand how to live with the illness, to learn how to face the critical moments of the clinical course, and to live in harmony with all health professionals. In addition, there may be several advantages for health professionals: a reduction in emotional labour, increased professional satisfaction, and a reduction in the potential tensions and conflicts with patients and their relatives. We suggest that therapeutic patient education in oncology may be useful for both patients and health professionals and probably lead to a reduction in the costs of healthcare delivery.

Keywords: oncology, education, health, prevention, therapy.

\section{INTRODUCTION: EDUCATION TO HEALTH AND PREVENTION}

According to the World Health Organization (WHO), chronic diseases have the highest prevalence (about $80 \%$ of total patients); of these patients, less than $50 \%$ usually follow the prescribed treatments. A recent definition by the WHO (1998) describes the so-called 'therapeutic patient education' (TPE) as an approach to support patients and their families to better understanding of their

Correspondence address: Manfredi Rizzo, Department of Clinical Medicine and Emerging Diseases, University of Palermo, Via del Vespro, 141, 90127, Palermo, Italy (e-mail: mrizzo@unipa.it).

Accepted 3 July 2006

DOI: $10.1111 / j .1365-2354.2006 .00716 . x$

European Journal of Cancer Care, 2007, 16, 9-11 illness, with the therapeutic options. There is a clear and specific difference between the informal-type patient education and the TPE, as conducted by the pedagogical criteria and methods: the former mainly consists of the patient-physician relation, with the prescriptions by the doctors of information, suggestions, advice, recommendations and instructions; and the latter consists of a training programme to enable patients to practise themselves, rather than only rely on doctors.

Since TPE needs a high level of communications between patients and health professionals, it cannot be applied to any patient, but should be individualized (Langton et al. 2003). Moreover, TPE represents a complex training, articulated in multiple steps, from the 'educational diagnosis' to the identification of the educational targets, with different therapeutic choices (World Health Organization 1998) (Table 1). 
Table 1. Therapeutic patient education: an example of systematic approach

- Identifying the patient's educational needs ('educational diagnosis')

- Exchanging educational contents and objectives with the patient ('educational-therapeutic contract')

- Suggesting alternative and interacting teaching approaches ('active teaching/learning')

- Checking educational activity quality and results by qualified methods ('evaluation')

\section{THERAPEUTIC PATIENT EDUCATION IN ONCOLOGY}

In Italy, cancers represent the second cause of death (about $1 / 3$ of all causes of death), after cardiovascular disease. In Italy, the annual incidence of cancer is about 250000 and the mortality rate is 150000 ; in addition, every year more than 1 million patients receive therapies for their oncology diseases in our hospitals (Verdecchia et al. 2001). The presence of a cancer involves not only the patient but the whole family, with a high emotional impact on their everyday life. The view of a suffering future is often devastating for the psycho-physical equilibrium of both patients and their relatives. Even if patients recover from cancer initially, regular checks are needed to detect any recurrence; all these aspects highly influence the patient's quality of life.

In 1998, the WHO provided a list of diseases that may benefit by the development of activities of TPE. This list included cancer, but its use in the oncology field is still very rare (Maung et al. 1993; Depondt \& Gehanno 1995; De Wit et al. 1997; Grahn et al. 1999). There may be several reasons for this. In Latin countries, compared with the USA for example, talking about cancer is less frequent (Surbone \& Zwittner 1997), and in Italy, informing patients of a diagnosis of cancer is still rare (Marcolongo et al. 2001; Verdecchia et al. 2001).

The diagnosis of cancer frequently has a negative impact on patients. They may be defensive, in denial and losing interest in the future. Patients often live in a style of chronic crisis. In this context, it is often difficult to plan a programme of TPE (Fahrenfort 1987; Demin 1997). However, cancer does not represent only one disease but many, and the TPE may not always be useful. About $15 \%$ of cancers lead to recovery (e.g. Hodgkin lymphoma, cancers of thyroid and testicle, and child tumours), but others lead to death (e.g. lung, pleura, stomach, liver and pancreas cancer). In addition, there are tumours that do not lead to a complete recovery but have a long period of survival (e.g. non-Hodgkin lymphoma, and chronic leukaemia of breast, intestine and bladder), during which patients have to learn to live with their cancer.
Table 2. An example of educational approach in oncology

Areas of educational intervention

- Primary and secondary prevention

- Post-surgery recovery

- Patient's everyday life (nutrition, physical and sexual activity, job, spare timel

- Emotional sphere

- Management of the clinical follow-up (keeping a personal clinical diary, booking laboratory analysis or doctors' checkup visits|

Specific aims

- Improving the quality of life of patients and their relatives

- Improving the quality of the clinical course

- Promoting a model of collaboration between all health professionals

- Promoting harmonic human relations between all subjects involved in the programme

The methodology of the educational-therapeutic interventions

- Constant adhesion to subjects' needs

- Relevancy of the learning objectives

- Economy, convenience, realism

- Suitably, brightness

- Active subjects' involving

- Strictness, independence

- Periodical and systematic evaluation of the educational programme

TPE may then be more appropriate. Therefore, besides the definition of the clinical problems, specific approaches of educational diagnosis may be made to explore the psycho-affective (who is?), social-professional (what does he do?) and knowledge (what does he know?) spheres. It is also useful to quickly involve their relatives, since patients' grade of learning is strictly linked to the grade of acceptance of their own illness.

\section{EDUCATIONAL APPROACHES IN ONCOLOGY}

We suggest that any educational approach should be preceded by the identification of the potential areas of intervention, with the specific learning aims and objectives to be achieved and the methodology to be used (Table 2).

In order to plan the activities of TPE, all health professionals should revise their medical, pedagogical and psychological notions (Block et al. 2004; Schofield \& Butow 2004). Therefore, the first goal to be reached is the formation of a specific working group, which may start and coordinate the programme. Moreover, within the working group, it is necessary to define the specific competence and roles for each professional (physicians, psychologists, nurses, volunteers and professional educators).

The involvement of physicians remains a problem. They do not see it as a priority in their work. In an example, psychologists are the main operators for the promotion and supervision of this education, particularly with reference to the emotional aspects. Nurses may be dedicated to 
ensuring that the education is available for both patients and their relatives. The volunteers are supportive, providing that they are included and well informed. Professional educators are new in the clinical medicine context in Italy, but they may cooperate in the projection, management and evaluation of the educational interventions.

\section{CONCLUSIONS}

Therapeutic patient education has been defined by the WHO as a comprehensive approach to support patients and their families to better understanding of their diseases. There is a clear and specific difference between the informal-type patient education and the TPE, as conducted by the pedagogical criteria and methods: the former mainly consists of the patient-physician relation, while the latter consists of a training programme to enable patients to practise themselves, rather than only rely on doctors.

In oncology, the contribution of therapeutic education may enable the patients to have adequate information of the illness, to actively participate in the management of the disease, to understand how to live with the illness, to learn how to face the critical moments of the clinical course and to live in harmony with all health professionals. In addition, there may be several advantages for health professionals: a reduction in emotional labour, increased professional satisfaction, and a reduction in the potential tensions and conflicts with patients and their relatives. We suggest that the TPE in oncology may be useful for both patients and health professionals and probably lead to a reduction in the costs of healthcare delivery.

\section{REFERENCES}

Block K.I., Block P. \& Gyllenhaal C. (2004) The role of optimal healing environments in patients undergoing cancer treatment: clinical research protocol guidelines. Journal of Alternative and Complementary Medicine 10, S157-S170.

De Wit R., van Dam F., Zandbelt L., van Buuren A., van der Heijden K., Leenhouts G. \& Loonstra S. (1997) A pain education program for chronic cancer pain patients: follow-up results from a randomized controlled trial. Pain 73, 55-69.

Demin E. (1997) Communication with the breast-cancer patient. An opinion from Russia. Annals of the New York Academy of Sciences 809, 485-495.

Depondt J. \& Gehanno P. (1995) Laryngectomized patients' education and follow-up. Patient Education and Counselling 26, 33-36.

Fahrenfort M. (1987) Patient emancipation by health education, an impossible goal? Patient Education and Counselling 10, 2537.

Grahn G., Danielson M. \& Ulander K. (1999) Learning to live with cancer in European countries. Cancer Nursing 23, 213221.

Langton H., Barnes M., Haslehurst S., Rimmer J. \& Turton P. (2003) Collaboration, user involvement and education: a systematic review of the literature and report of an educational initiative. European Journal of Oncology Nursing 7, 242252.

Marcolongo R., Rossato E., Pavan V., Laveder F., Bonadiman L. \& Rigoli A.M. (2001) Current perspectives of therapeutic patient education in Italy. Patient Education and Counselling 44, 59-63.

Maung Z.T., Taylor P.R., Robinson P., Moore J., Lucraft H.H., Evans R.G. \& Proctor S.J. (1993) Patient education for selfreferral and on-demand treatment for herpes zoster in lymphoma patients. Leuk Lymphoma 11, 447-452.

Schofield P.E. \& Butow P.N. (2004) Towards better communication in cancer care: a framework for developing evidencebased interventions. Patient Education and Counselling 55, 32-39.

Surbone A. \& Zwittner M. (1997) Communication with the cancer patient: information and truth. Annals of the New York Academy of Sciences 809, 1-6.

Verdecchia A., Mariotto A., Capocaccia R., Gatta G., Micheli A., Sant M. \& Berrino F. (2001) Incidence and prevalence of all cancerous diseases in Italy: trends and implications. European Journal of Cancer 37, 1149-1157.

World Health Organization (WHO) (1998) Therapeutic Patient Education, Continuing Education Programs for Healthcare Providers in the Field of Prevention of Chronic Diseases. Report of a WHO working group, Regional Office for Europe, Geneva. 